\title{
CHEMPLUSCHEM
}

Supporting Information

(C) Copyright Wiley-VCH Verlag GmbH \& Co. KGaA, 69451 Weinheim, 2019

\section{Supramolecular Gelation of Europium and Calcium Cholates through the Nucleation-Elongation Growth Mechanism}

Raju Laishram and Uday Maitra* 
Table SI1. IR data of $\mathrm{NaCh}, \mathrm{CaCh}$ and EuCh lyophilized samples.

\begin{tabular}{lll}
\hline Samples & $\begin{array}{l}v_{\mathrm{c}=0} \text { (Symmetric stretching) } \\
\mathrm{cm}^{-1}\end{array}$ & $\begin{array}{l}v_{\mathrm{c}=0} \text { (Asymmetric stretching) } \\
\mathrm{cm}^{-1}\end{array}$ \\
NaCh & 1401 & 1555 \\
CaCh sol & 1411 & 1551 \\
CaCh gel & 1405 & 1550 \\
EuCh sol & 1408 & 1548 \\
EuCh gel & 1405 & 1542
\end{tabular}

\section{TEM Imaging}
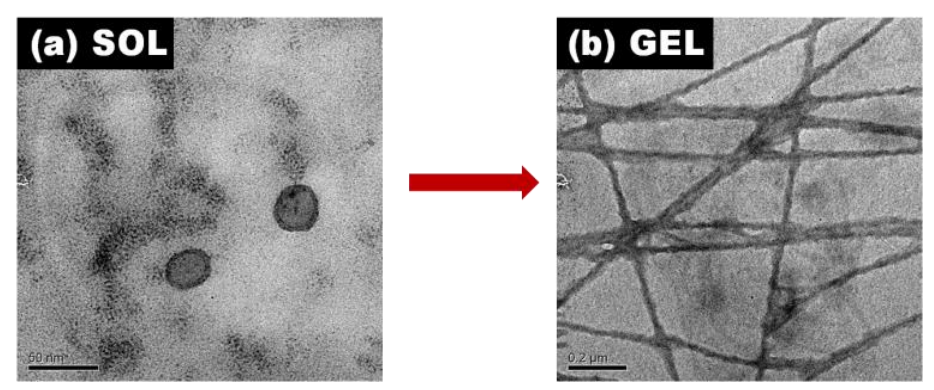

Fig. SI1 TEM of CaCh (lyophilized sample; 15/30 mM): (a) solution (just after mixing) and (b) gel (after 3 h)

TEM images (SI1) of CaCh gave similar results as the AFM (Fig. 2). The particles were observed to be $\sim 30 \mathrm{~nm}$ in the sol phase. After gel formation, a fibrous network with fibre diameter of $\sim 30 \mathrm{~nm}$ was seen which was similar to the diameter of the particles in the sol phase.

\section{Confocal Imaging}

The morphology changes were also studied using fluorescence confocal imaging. Rhodamine 6G was used as the fluorescence dye. The imaging was done just after mixing and also after 3 hrs. The resolution of confocal is 200 $\mathrm{nm}$ so individual particles or fibres cannot be resolved. However, clear transition from particle-like structures, in sol phase, to fibres like morphology. In gel phase, was observed.

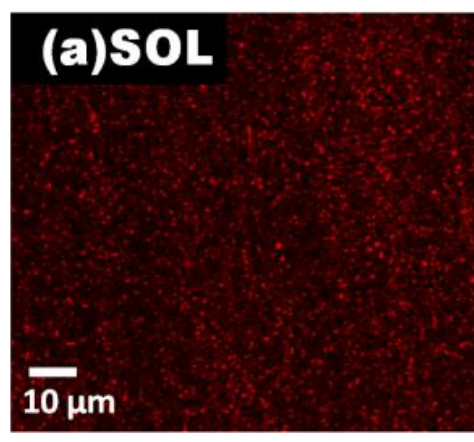

\section{(b) GEL}


Fig SI2 Fluorescence confocal image of CaCh: (a) solution and (b) gel

EuCh gelation was fast and we were unable to get the TEM and Confocal images of sol phase having particles, we got only fibrous network. However, the AFM images prove the particles to fibre transition in sol to gel. This was because the hydrophobic nature of the carbon coated copper grid used in TEM imaging accelerates the aggregation process, whereas the hydrophilic nature of the mica sheet used for AFM imaging aggregation slows down the aggregation. In confocal imaging, the imaging took more time ( 10-30 min) and, moreover, we cannot freeze the sample at the sol phase. Thus, for fast gelling EuCh, we were not able to observe the sol phase, only EuCh gel phase was observe (SI4).

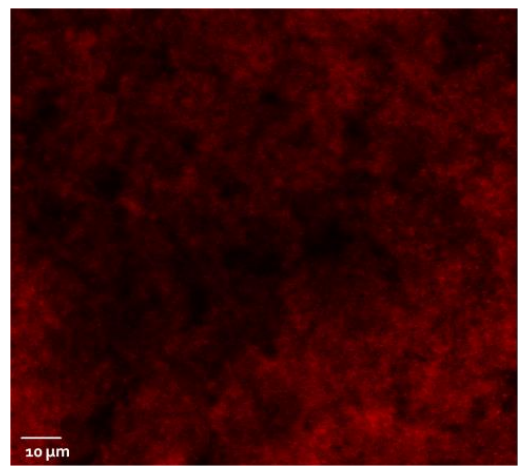

Fig. SI3 Fluorescence confocal image of EuCh gel

\section{Fluorescence of $\mathrm{Eu}(\mathrm{III})$ in $\mathrm{EuCh}$ in $\mathrm{D}_{2} \mathrm{O}$}
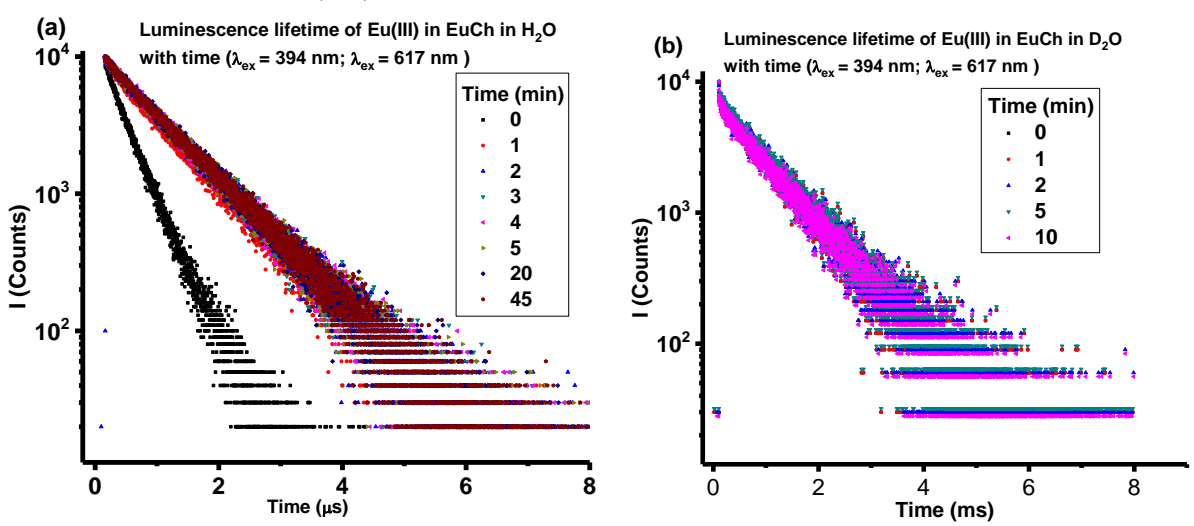

Fig. SI4 Luminescence lifetime of EuCh with time in (a) $\mathrm{H}_{2} \mathrm{O}$ and(b) $\mathrm{D}_{2} \mathrm{O}$ 


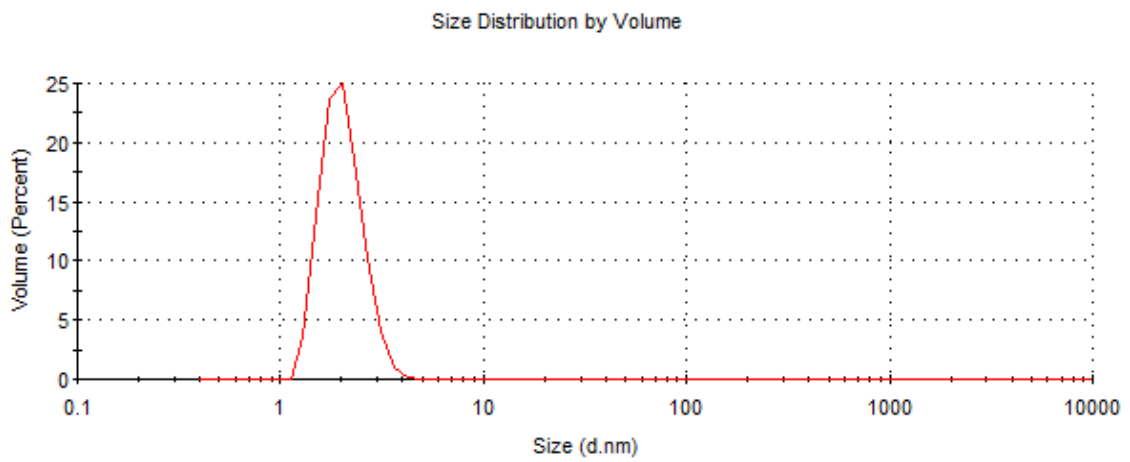

Fig. SI5 DLS of CaCh $(5 / 15 \mathrm{mM}) \mathrm{sol}$ 